\title{
Impact of Urbanization on Detection Rates of Eating Disorders
}

\author{
Hans W. Hoek, M.D., Ph.D., Aad I.M. Bartelds, M.D., Jacquoline J.F. Bosveld, M.A., \\ Yolanda van der Graaf, M.D., Ph.D., Véronique E.L. Limpens, M.A., \\ Margo Maiwald, M.D., and Caroline J.K. Spaaij, Ph.D.
}

\begin{abstract}
Obiective: The purpose of this study was to examine the incidence of anorexia nervosa and bulimia nervosa among patients in primary care and to evaluate the impact of urbanization, age and sex differences, and changes over time. Method: During 1985-1989, 58 general practitioners, trained in diagnosing eating disorders, registered all of their patients who had diagnoses of anorexia nervosa and/or bulimia nervosa according to strict criteria. The study population $(N=151,781)$ was $1 \%$ of the population of the Netherlands; the distribution of sexes, ages, geographical locations, and degrees of urbanization in the study group was representative of the Dutch population. Main outcome measures were rates of newly detected cases and age-adjusted rate ratios. Results: The crude annual incidence rate of detected cases in primary care per 100,000 person-years was 8.1 for anorexia nervosa and 11.5 for bulimia nervosa. The incidence of bulimia nervosa was lowest in rural areas, intermediate in urbanized areas, and highest in large cities (6.6,19.9, and 37.9, respectively, per 100,000 females per year); no rural-urban differences for anorexia nervosa were found. Pronounced sex and age differences in incidence rates were observed. Over the 5-year period, there was no time trend in the incidence of anorexia nervosa, but the incidence of bulimia nervosa tended to increase. Conclusions: The incidence rates of eating disorders-as defined by detection rates in primary care-are higher than previously reported. Urbanization seems to be a risk factor for bulimia nervosa but not for anorexia nervosa.

(Am J Psychiatry 1995; 152:1272-1278)
\end{abstract}

$\mathrm{E}$ pidemiological research on eating disorders has hitherto consisted merely of determining the prevalence of anorexia nervosa and bulimia nervosa within populations and comparing the prevalence in different population groups $(1,2)$. Most epidemiological studies have focused on the prevalence of eating disorders in the population most at risk: females aged 10-30 years. The more sophisticated studies, which were two-stage surveys based on strict diagnostic criteria, showed that the prevalence of eating disorders among young females in the community is about $0.3 \%$ for anorexia nervosa and about $1 \%$ for bulimia nervosa $(2,3)$.

The limited knowledge about the pathogenesis of anorexia nervosa has recently been described by Garner

Received July 5, 1994; revision received Dec. 27, 1994; accepted March 31, 1995. From the Department of Psychiatry and the Department of Epidemiology, University Hospital Utrecht, and the Netherlands Institute of Primary Health Care, Utrecht. Address reprint requests to Dr. Hoek, Psychiatric Hospital Rosenburg, P.O. Box 53019, 2505 AA The Hague, The Netherlands.

Supported by the Dutch Ministry of Health.

The authors thank the general practitioners for participating in the study and Prof. Dr. René S. Kahn, Prof. Dr. Herman van Engeland, and Dr. Michael King for critically reading the manuscript.

(4). To help us understand more about the etiology of eating disorders, we need incidence studies. The incidence rate is defined as the number of new cases in the population per year, whereas the prevalence rate refers to the actual number of cases in a population at a certain point in time. So far, incidence studies of anorexia nervosa have been based either on psychiatric case registers $(5-12)$ or on medical records of hospitals in a circumscribed area (13-15). Psychiatric case registers contain cases treated in mental health care, whereas medical record studies identify hospital inpatients, including those admitted to psychiatric, medical, and pediatric wards. The reported incidence varies considerably, from less than one case up to five cases per year per 100,000 of the total population. Overall, these studies show an increase in the registered incidence of anorexia nervosa over time. However, the studies disagree about whether there was an increase in the ageadjusted incidence of anorexia nervosa in the $1980 \mathrm{~s}$ compared to the 1970s $(9-12,14,15)$. Most cases of anorexia nervosa occur in women, but $5 \%-10 \%$ of persons presenting for treatment are men (2).

There have been two studies that tried to estimate the incidence of bulimia nervosa; both of them had methodological limitations. Cullberg and Engström- 
Lindberg (16) asked secondary and primary care personnel about the possible cases of eating disorders they encountered during 1984-1985. They estimated the "1-year incidence rate" for bulimia nervosa to be 3.9 per 100,000 population, but this figure was derived from only six cases. Joergensen (12) studied the incidence in Fyn County, Denmark, using national and local registers; these registers recorded only possible diagnoses of anorexia nervosa, not of bulimia nervosa. Through rediagnosis of the cases registered as anorexia nervosa, he estimated the admission rate for bulimia nervosa to be 5.5 per year per 100,000 females aged 10-24 years.

The incidence derived from case registers and medical record studies relates to incidence of treated cases and does not necessarily reflect the incidence in the community. To find more accurate estimates of community rates, we decided to study the incidence of anorexia nervosa and bulimia nervosa in primary care. Primary care physicians generally have difficulty in detecting eating disorders, because patients often deny or conceal their illness. However, if general practitioners are trained in diagnosing eating disorders, they will often be able to detect a physical sign or symptom of an eating disorder when patients consult them for some other problem. General practitioners can also obtain information about a patient from health care workers such as medical specialists, who in the Netherlands are obliged to inform general practitioners of their findings. They may also be informed indirectly through observations and comments made by worried family members.

Prevalence data from our primary care study over the 1985-1986 period have been published elsewhere (17). Incidence figures were presented in that paper, but at that stage the number of incident cases was too limited to compare incidence rates in subgroups. It was found that the prevalence of bulimia nervosa is three times higher in large cities than in smaller urbanized or rural areas, whereas anorexia nervosa occurred with almost equal frequency in areas with different degrees of urbanization. In that study it was suggested that the difference in the prevalence of the two disorders might be explained in terms of a "drift" hypothesis; i.e., bulimia nervosa patients are somewhat older than anorexia nervosa patients-most of them being between 20 and 29 years old, an age group that is more likely to move to cities than the younger anorexic patients. However, a possible age effect could not yet be investigated. The substantial number of incident cases in the current study allows us to evaluate the drift hypothesis with age-adjusted rate ratios by degree of urbanization.

The purpose of the current study was to answer the following questions.

1. What is the incidence of anorexia nervosa and bulimia nervosa in primary care?

2. Is there a difference between cities, urbanized areas, and rural areas in the age-adjusted incidence of eating disorders?

3. Does the incidence of eating disorders change over time?
4. What is the difference in incidence of eating disorders between males and females?

5. What are the age-specific incidence rates of eating disorders in primary care?

\section{METHOD}

Since 1970 , continuous morbidity registration has been performed in a Dutch network of sentinel stations by 58 general practitioners (18). These general practitioners are experienced in registering diseases, and every year they record data on a few selected disorders. In 1985 these practices together served an average of 151,781 people (1.1\% of the Dutch population), and in 1989 they served 144,872 people.

The total population under study has been divided into three urbanization groups according to the typology of the National Institute of Statistics: rural areas $(20 \%$ or more of the population in agrarian labor), urbanized areas, and large cities (more than 100,000 inhabitants). Urbanized areas include urbanized rural municipalities, municipalities with urban characteristics, and small cities (fewer than 100,000 inhabitants). In 1985 there were 23,470 people living in rural areas, 91,428 people in urbanized areas, and 36,883 people in large cities. Expressed as a percentage of the total Dutch population in each of the three urbanization areas, this study population comprised $1.4 \%$ of the rural population, $1.0 \%$ of the urbanized population, and $1.1 \%$ of the large-city population. Thus, the spread of the study population over the three urbanization categories was representative of the total Dutch population, with a slight overrepresentation of people in rural areas. The population under study was also representative of the Dutch population with respect to sex, age, and geographical spread (18).

The participating general practitioners registered the incidence of anorexia nervosa and bulimia nervosa from January 1985 until the end of December 1989. Annually, they received detailed information on eating disorders by means of a circular and at meetings convened for the purpose. The general practitioners considered whether each patient who consulted them might possibly be suffering from anorexia nervosa or bulimia nervosa. Sometimes the general practitioner was alerted to the possibility of an eating disorder by other health care workers or worried relatives. The criteria for both disorders (appendix 1) were derived from the DSM-III classification and the criteria proposed in 1985 that led to DSM-III-R (published in 1987).

To qualify as a "case," a patient had to meet all three criteria in appendix 1 for either anorexia nervosa or bulimia nervosa. If the general practitioner doubted whether a possible case met the criteria, two experts in eating disorders (H.W.H. and M.M.) made the decision, taking into account any additional information the general practitioner could give. Of the total number of 178 possible cases, 40 patients with an eating disorder were excluded from the study because the evidence was not conclusive.

To comply with a DSM-III-R (and DSM-IV) case definition of anorexia nervosa, the patient had to meet the three criteria in appendix 1 and also to have suffered from amenorrhea for a period of at least 3 months. To comply with a DSM-III-R case definition of bulimia nervosa, the three criteria in appendix 1 had to be met and the patient also had to have had a minimum average of two binge-eating episodes a week for a period of at least 3 months.

The incidence rate was defined as the number of new cases in primary care per year. It was based on the time when the eating disorder was detected, because obviously it was impossible to know precisely when the eating disorder began. All patients detected before 1985 and still exhibiting features of the disorder in 1985 were considered to be "old" cases. Only patients with an eating disorder detected during 1985-1989 were considered incident cases. The incidence rate was calculated by dividing the number of detected incident cases by the number of person-years of the population under study; $95 \%$ confidence intervals were based on the Poisson distribution (19). If the $95 \%$ confidence interval included 1 , the results were considered to be nonsignificant. The age-adjusted rate ratios for estimating the effect of living in areas with different degrees of urbanization on the risk of 
TABLE 1. Incidence Rates of Anorexia Nervosa and Bulimia Nervosa per 100,000 Person-Years in Primary Care in the Netherlands, 1985-1989

\begin{tabular}{|c|c|c|c|c|c|c|c|}
\hline \multirow[b]{2}{*}{ Year } & \multicolumn{3}{|c|}{ Anorexia Nervosa } & \multicolumn{3}{|c|}{ Bulimia Nervosa } & \multirow[b]{2}{*}{ Person-Years } \\
\hline & $\mathbf{N}$ & $\begin{array}{c}\text { Incidence } \\
\text { Rate }\end{array}$ & $\begin{array}{c}95 \% \text { Confidence } \\
\text { Interval } \\
\end{array}$ & $\mathrm{N}$ & $\begin{array}{c}\text { Incidence } \\
\text { Rate }\end{array}$ & $\begin{array}{c}95 \% \text { Confidence } \\
\text { Interval }\end{array}$ & \\
\hline 1985 & 12 & 7.9 & $4.5-13.9$ & 11 & 7.2 & $4.0-13.1$ & 151,781 \\
\hline 1986 & 7 & 4.6 & $2.2-9.8$ & 19 & 12.6 & $8.0-19.8$ & 150,557 \\
\hline 1987 & 12 & 8.1 & $4.6-14.3$ & 15 & 10.1 & $6.1-16.8$ & 148,001 \\
\hline 1988 & 11 & 7.5 & $4.2-13.6$ & 18 & 12.3 & $7.7-19.5$ & 146,509 \\
\hline 1989 & 18 & 12.4 & $7.8-19.7$ & 22 & 15.2 & $10.0-23.1$ & 144,872 \\
\hline $1985-1989$ & 60 & 8.1 & $6.1-10.2$ & 85 & 11.5 & $9.0-13.9$ & 740,091 \\
\hline
\end{tabular}

TABLE 2. Crude and Age-Adjusted Incidence Rate Ratios of Anorexia Nervosa and Bulimia Nervosa by Degree of Urbanization in Females in the Netherlands, 1985-1989

\begin{tabular}{|c|c|c|c|c|c|c|c|c|}
\hline \multirow[b]{2}{*}{ Ratio } & \multicolumn{4}{|c|}{ Anorexia Nervosa } & \multicolumn{4}{|c|}{ Bulimia Nervosa } \\
\hline & $\begin{array}{c}\text { Crude } \\
\text { Rate } \\
\text { Ratio }\end{array}$ & $\begin{array}{c}95 \% \\
\text { Confidence } \\
\text { Interval }\end{array}$ & $\begin{array}{c}\text { Age- } \\
\text { Adjusted } \\
\text { Rate Ratio }\end{array}$ & $\begin{array}{c}95 \% \\
\text { Confidence } \\
\text { Interval }\end{array}$ & $\begin{array}{c}\text { Crude } \\
\text { Rate } \\
\text { Ratio }\end{array}$ & $\begin{array}{c}95 \% \\
\text { Confidence } \\
\text { Interval }\end{array}$ & $\begin{array}{c}\text { Age- } \\
\text { Adjusted } \\
\text { Rate Ratio }\end{array}$ & $\begin{array}{c}95 \% \\
\text { Confidence } \\
\text { Interval }\end{array}$ \\
\hline Urbanized areas to rural areas & 0.73 & $0.36-1.45$ & 0.75 & $0.38-1.49$ & 3.00 & $1.08-8.33$ & 2.99 & $1.08-8.31$ \\
\hline Cities to rural areas & 0.88 & $0.40-1.94$ & 0.96 & $0.44-2.13$ & 5.72 & $2.03-16.14$ & 5.48 & $1.94-15.48$ \\
\hline Cities to urbanized areas & 1.22 & $0.64-2.29$ & 1.29 & $0.68-2.44$ & 1.91 & $1.22-2.99$ & 1.83 & $1.17-2.88$ \\
\hline
\end{tabular}

developing an eating disorder were calculated by Poisson regression analysis (19) with use of the program EGRET, version 0.26.6 (SERC, Seattle, Wash.); this provides a good approximation of the binomial distribution for rare events.

The body mass index was calculated as body weight (in kilograms) divided by height (in meters squared). Values between 20 and 25 were considered to be normal (20).

\section{RESULTS}

Before 1985, 28 patients had been diagnosed as having anorexia nervosa and 31 patients as having bulimia nervosa, and they were still suffering from an eating disorder during 1985. This yielded a point prevalence of 18.4 per 100,000 population for anorexia nervosa $(95 \%$ confidence interval $=12.7-26.8$ ) and 20.4 for bulimia nervosa (95\% confidence interval $=14.4-29.1)$ (17).

In the period between 1985 and 1989, the general practitioners detected 138 new cases that definitely met our criteria for anorexia nervosa or bulimia nervosa. Seven of the patients met the criteria for both disorders. The newly detected cases involved 130 female and eight male patients.

\section{Incidence of Anorexia Nervosa}

During 1985-1989, 60 patients received a first diagnosis of anorexia nervosa, which yields an incidence of 8.1 per 100,000 person-years (table 1 ). By definition, all anorexia nervosa patients were underweight (body mass index <20). To meet the DSM-III-R (and DSM-IV) criteria, female patients also had to have missed at least three consecutive menstrual cycles. If we exclude those who did not have amenorrhea and those about whom information on current menstrual status was inaccurate, the crude incidence of anorexia nervosa (DSM-IIIR/DSM-IV classification) was 6.8 per 100,000 person- years $(95 \%$ confidence interval $=4.9-8.6)$. During the period $1985-1989,63 \%$ of the patients with anorexia nervosa were referred to mental health care, yielding an incidence in mental health care of 5.1 per 100,000 person-years.

The incidence rate of anorexia nervosa per year per 100,000 females was 18.2 in rural areas, 13.2 in urbanized areas, and 16.1 in large cities. The $95 \%$ confidence intervals of the age-adjusted rate ratios-which were almost similar to the crude rate ratios-each included 1 , meaning that after adjustment for the differences in age distribution, the incidence rates in the three urbanization categories were not significantly different from one another (table 2).

There was no trend over time in the incidence of anorexia nervosa during the period 1985-1989 (table 1).

Of all anorexia nervosa patients, five $(8.3 \%)$ were male (aged 12, 13, 16, 23, and 24 years). The incidence rate for males was 1.4 per year per 100,000 (95\% confidence interval=0.6-3.4). The incidence rate for females of all ages was 14.7 per 100,000 woman-years. Table 3 shows the age-specific incidence of anorexia nervosa for females during 1985-1989. The highest incidence, namely, 79.6 per 100,000 woman-years, was in the 15-19 age group.

\section{Incidence of Bulimia Nervosa}

During 1985-1989, 85 patients received a first diagnosis of bulimia nervosa, which yields an incidence of 11.5 per 100,000 person-years (table 1). Of all bulimia nervosa patients, $12(14.1 \%)$ were underweight (body mass index $<20), 49(57.6 \%$ ) were overweight (body mass index $>25$ ), and $24(28.2 \%)$ were of normal weight.

The general practitioners were unable to provide accurate information on binge-eating behavior for some patients, because these patients denied binge eating. In 
TABLE 3. Age-Specific Incidence Rates of Anorexia Nervosa and Bulimia Nervosa per Year per 100,000 Females in Primary Care in the Netherlands, 1985-1989

\begin{tabular}{|c|c|c|c|c|c|c|c|}
\hline \multirow[b]{2}{*}{ Age (years) } & \multicolumn{3}{|c|}{ Anorexia Nervosa } & \multicolumn{3}{|c|}{ Bulimia Nervosa } & \multirow[b]{2}{*}{ Woman-Years } \\
\hline & $\mathrm{N}$ & $\begin{array}{c}\text { Incidence } \\
\text { Rate }\end{array}$ & $\begin{array}{c}95 \% \text { Confidence } \\
\text { Interval } \\
\end{array}$ & $\mathrm{N}$ & $\begin{array}{c}\text { Incidence } \\
\text { Rate }\end{array}$ & $\begin{array}{c}95 \% \text { Confidence } \\
\text { Interval }\end{array}$ & \\
\hline $10-14$ & 2 & 8.6 & $2.2-34.4$ & 3 & 12.9 & $4.2-40.0$ & 23,245 \\
\hline $15-19$ & 24 & 79.6 & $53.4-118.7$ & 13 & 43.1 & $26.0-74.2$ & 30,155 \\
\hline $20-24$ & 11 & 33.4 & $18.5-60.4$ & 27 & 82.1 & $58.3-119.7$ & 32,900 \\
\hline $25-29$ & 12 & 37.9 & $21.5-66.7$ & 20 & 63.1 & $40.7-97.8$ & 31,700 \\
\hline $30-34$ & 3 & 10.2 & $3.3-31.7$ & 8 & 27.3 & $13.7-54.6$ & 29,300 \\
\hline $35-64$ & 3 & 2.3 & $0.7-7.0$ & 11 & 8.3 & $4.6-15.0$ & 133,055 \\
\hline $10-64$ & 55 & 19.6 & $14.4-24.8$ & 82 & 29.2 & $22.9-35.6$ & 280,355 \\
\hline All ages & 55 & 14.7 & $11.0-18.4$ & 82 & 21.9 & $17.2-26.7$ & 373,975 \\
\hline
\end{tabular}

these cases the general practitioner heard about frequent binges through the family. To meet the DSM-III$\mathrm{R}$ criteria for bulimia nervosa, the patient had to satisfy the criteria in appendix 1 and the additional criterion of a minimum average of two binge-eating episodes a week for at least 3 months. If we exclude the patients whose frequency of binge eating was not known with certainty, the incidence drops to 9.7 per 100,000 person-years $(95 \%$ confidence interval $=7.5-12.0)$.

The incidence rate for bulimia nervosa per year per 100,000 females was 6.6 in rural areas, 19.9 in urbanized areas, and 37.9 in large cities (with more than 100,000 inhabitants). Age-adjusted rate ratios were used to compare the incidence rates in the three urbanization categories, taking into account the (small) differences in age distribution (table 2 ). The $95 \%$ confidence intervals of these age-adjusted rate ratios did not include 1 , meaning that the incidence rates of bulimia nervosa in the three urbanization categories were significantly different from one another.

The incidence rates of bulimia nervosa tended to increase during 1985-1989. The incidence increased by $15 \%$ each year (rate ratio $=1.15 ; 95 \%$ confidence interval $=0.99-1.33$ ).

Fifty-three percent $(\mathrm{N}=45)$ of the bulimia nervosa patients were referred to mental health care. Most (39\%, $\mathrm{N}=33$ ) of the other patients were treated by the general practitioners themselves. During 1985-1989, the incidence of bulimia nervosa in mental health care was 6.0 per 100,000 person-years.

Of all bulimia nervosa patients, only three $(3.5 \%)$ were male (aged 14, 20, and 23 years). The incidence rate for males was 0.8 per year per $100,000(95 \%$ confidence interval $=0.3-2.6)$. The incidence rate for females of all ages was 21.9 per 100,000 woman-years (table 3 ). The highest incidence rate, namely, 82.1 per year per 100,000 females, was observed in the 20-24 age group.

\section{DISCUSSION}

In our study, the incidence of bulimia nervosa in primary care was 11.5 per 100,000 person-years. Our incidence figure decreases to 9.7 per 100,000 personyears if our case definition includes the additional certainty criterion regarding a minimum average of two binge-eating episodes per week for at least 3 months (DSM-III-R). In the DSM-IV classification, the diagnosis of bulimia nervosa cannot be given if anorexia nervosa is present. With the use of this additional criterion, the incidence of bulimia nervosa would decrease to 9.1 per 100,000 person-years.

The incidence rate for anorexia nervosa in primary care was 8.1 per 100,000 person-years in our study, which is much higher than that found in psychiatric case registers or medical record studies, except for the study by Lucas et al. (15). Including probable and possible cases, Lucas et al. found the incidence of anorexia nervosa to be 8.2 per 100,000 person-years, which is comparable to our figure. They screened not only the records of patients with a clinical diagnosis of anorexia nervosa but also the records of patients with amenorrhea, oligomenorrhea, starvation, weight loss, or some other related diagnosis. The incidence in our study decreases to 6.8 per 100,000 person-years - still a high rate-if we include amenorrhea as an additional certainty criterion for case definition (DSM-III-R and DSM-IV).

The incidence rates relate to persons who consulted their general practitioners for any kind of medical problem and to persons regarding whom the general practitioners received information from some other source. Our figures depend on the detection skills of general practitioners. On the whole, general practitioners have difficulty in detecting eating disorders $(21$, 22). In a British study of community subjects with bulimia nervosa (23), just $10 \%$ were in treatment. However, the general practitioners in our study already had experience in registering other kinds of disorders and had also received written and oral instructions about the detection of eating disorders. Although the general practitioners in our study were better informed about eating disorders than most other general practitioners, they would inevitably have missed some cases, because eating disorders are characterized by taboo and denial. Because they are underweight, patients with anorexia nervosa are more easily detected than patients with bulimia nervosa. As a result of this, incidence rates for cases of anorexia nervosa are probably more reliable than those for bulimia nervosa. There is a surprising 
similarity of incidence rates of the two disorders in primary care, considering that community studies suggest that the prevalence of bulimia nervosa is threefold to fourfold higher than of anorexia nervosa (1-3). The relatively low number of bulimia nervosa patients with normal weight - the most common condition in clinical samples-might suggest that general practitioners have difficulty in detecting this specific condition. It is also possible that the general population-in contrast to clinical samples-comprises more overweight bulimia nervosa patients; somewhat similar results have been found in the United States (24). Our figures can be used as minimum estimates of the true incidence, namely, the incidence in the community. Our incidence figures are based on the time of detection and not on the time of onset. The age distribution will certainly be inflated by the difference between age at onset and age at detection.

Anorexia nervosa and bulimia nervosa have many similar characteristics and sometimes occur in the same person, either at the same time or at different times in life. The most striking result of our study is that the association between incidence and urbanization is different for anorexia nervosa than for bulimia nervosa. The crude incidence of bulimia nervosa was more than five times higher in large cities than in rural areas. A higher prevalence in urban than in rural areas has also been observed for other psychiatric disorders (schizophrenia and major depression) (25-27); however, rural-urban differences appear to be less pronounced for these disorders than for bulimia nervosa. There appeared to be no relation between anorexia nervosa and the degree of urbanization.

The difference between anorexia nervosa and bulimia nervosa with respect to the association between urbanization and detection rates could be related to the difference in age distribution. Bulimia nervosa patients are more often between the ages of 20 and 24 years, an age group in which many go to universities or other centers of advanced education, which in the Netherlands are mainly situated in large cities. However, after adjustment for age, pronounced differences in the detection rates of bulimia nervosa between rural areas, urbanized areas, and large cities were still observed. Thus, ruralurban differences in the incidence of bulimia nervosa are not caused by differences in age distribution. We therefore reject the drift hypothesis.

There might be a causal relationship between living in large cities and the development of bulimia nervosa. We only know rates of detected cases, and we do not know how much these rates reflect true incidence rates. Therefore, one has to be careful about drawing conclusions. It is possible that the general practitioners in the cities were, for unknown reasons, more alert to the diagnosis of bulimia nervosa than the general practitioners outside the cities. However, there is no reason to believe that a bias was introduced through systematic differences in case detection between general practitioners from different urbanization categories. Because Holland is a small country and has only eight medical schools (of similar rank), all general practitioners are considered to have had almost the same training. Hardly any area can be characterized as deprived. Annually, all general practitioners in the study received the same detailed information on eating disorders.

In speculating about the possibility of urbanization as a risk factor for bulimia nervosa, we can consider the possibility that in cities, increased pressure to be slender might be a causal factor. However, no relation between anorexia nervosa and degree of urbanization was found. One could argue that pressure to be slim is not of equal importance in the two disorders. If anorexia nervosa has a much more biological origin and is more driven by other factors such as a tendency toward asceticism and compulsive behavior, it may be less sensitive to social factors than is bulimia nervosa. Generally, people in rural areas are more likely to live with their families, and those in urban areas to be more independent and, perhaps, socially isolated. It could be that this exposes people in cities to more psychosocial stress and also gives them more opportunity to engage in a secretive behavior such as bulimia nervosa. Another factor with a possible causal relation might be the abundance in large cities of stimuli that provoke binge eating-for example, billboards, fast-food restaurants, and supermarkets. Yet another possibility is that in cities there is an unknown biological risk factor-e.g., nutrition or pollution-for developing bulimia nervosa.

Since the study covered only a relatively short period of 5 years (1985-1989), it is not surprising that we did not find a trend in the incidence of anorexia nervosa (table 1). To evaluate further the possibility of an increase in incidence, we compared the number of anorexia nervosa patients referred to mental health care as observed in this study in 1985-1989 (5.1 per year per 100,000 ) with the incidence found with the use of a psychiatric case register during 1974-1982 (5.0 per 100,000 person-years) (7). The psychiatric case register included all inpatients and outpatients with anorexia nervosa in Assen in the Netherlands. This comparison did not indicate any increase in the incidence of anorexia nervosa either. While there seems to have been an increase in the incidence of anorexia nervosa among young females in the last 50 years (15), our study suggests that there was no increase in the incidence of anorexia nervosa in the 1980s compared to the 1970s.

Recently, a Danish study based on records for 19701989 (11) found a significantly increasing incidence of first admissions in which anorexia nervosa was the main diagnosis. Studies of psychiatric inpatients in England from 1972 to 1981 (9) and in Denmark from 1973 to 1987 (10) showed that the number of first admissions for women with anorexia nervosa increased over time. However, analysis of age-period cohorts in these studies showed that this was due to an increase in the number of young women rather than to an elevated morbidity risk $(9,10)$. We therefore conclude that at present, no convincing evidence is available to indicate 
an increasing risk of anorexia nervosa during the 1980s compared to the 1970 s.

Our data for bulimia nervosa suggest an increase in the incidence of bulimia nervosa over the period 19851989. It is conceivable that since anorexia nervosa is harder to hide and has been known for a longer period of time, it is probably easier to detect. Since bulimia nervosa has only been considered a disease entity for a short period-beginning in 1980-its apparent increase may also be the result of increased awareness of the disorder among the general practitioners participating in the study (training effect) or of less taboo surrounding bulimia nervosa among patients, family members, and physicians.

Among males, bulimia nervosa appears to be even more rare than anorexia nervosa: $8 \%$ of all anorexia nervosa patients were male, whereas for bulimia nervosa, less than $4 \%$ of the patients were male. The differences in incidence rates between males and females might be partly the result of a higher risk of missing cases in males compared with cases in females. Detection of male patients might be more difficult than detection of female patients because of sex differences in the description of concerns about body shape and size and in the reasons for dieting (28). Still, for anorexia nervosa, the observation that $8 \%$ of the cases involved males fits well with earlier observations (1-3).

Incidence rates for bulimia nervosa in males have not been reported before. The lower incidence of bulimia nervosa versus anorexia nervosa in males contrasts with the higher incidence of bulimia versus anorexia nervosa in females. This observation might describe true differences, but it could also be the result of a less successful detection of bulimia nervosa in males than in females.

Eating disorders occur mostly, but not exclusively, in adolescents and young adults (table 3 ). The peak incidence for anorexia occurred in the age group of 15-19 years, which was also reported in earlier publications $(10-12,15,16)$. For bulimia nervosa, the incidence rate was highest in the age group of 20-24 years, but the peak was less pronounced and incidence figures were relatively high from 15 to 29 years. In addition to identifying the most vulnerable age groups, our study shows that both syndromes also occur (although rarely) in older women.

\section{CONCLUSIONS}

The incidence rates for anorexia nervosa and bulimia nervosa in our study are higher than those in previous studies and are probably better estimates of the occurrence at the community level. Both syndromes are seen predominantly in young women but do occur in older women and in men. The incidence of bulimia nervosa is highest in large cities, intermediate in urbanized areas, and lowest in rural areas. The degree of urbanization apparently has no impact on the incidence of anorexia nervosa.

\section{APPENDIX 1. Criteria for Case Identification of Anorexia Nervosa and Bulimia Nervosa (Based on DSM-III-R)}

Anorexia nervosa

1. Intense fear of becoming obese, even when underweight

2. Disturbance in the way in which one's body weight, size, or shape is experienced, e.g., claiming to "feel fat" even when emaciated, believing that one area of the body is "too fat" even when obviously underweight

3. Refusal to maintain body weight over a minimal normal weight for age and height, e.g., weight loss leading to maintenance of body weight $15 \%$ below expected weight; failure to make expected weight gain during the period of growth, leading to body weight $15 \%$ below expected weight

Bulimia nervosa

1. Recurrent episodes of binge eating (rapid consumption of a large amount of food in a discrete period of time, usually less than 2 hours)

2. During the eating binges, a feeling of lack of control over the eating behavior

3. Regular self-induced vomiting, use of laxatives, or rigorous dieting or fasting in order to counteract the effects of the binge eating

\section{REFERENCES}

1. Hsu LKG: Eating Disorders. New York, Guilford Press, 1990

2. Hoek HW: Review of the epidemiological studies on eating disorders. Int Rev Psychiatry 1993; 5:61-74

3. Fairburn CG, Beglin SJ: Studies of the epidemiology of bulimia nervosa. Am J Psychiatry 1990; 147:401-408

4. Garner DM: Pathogenesis of anorexia nervosa. Lancet 1993; 341: 1631-1635

5. Kendell RE, Hall DJ, Hailey A, Babigian HM: The epidemiology of anorexia nervosa. Psychol Med 1973; 3:200-203

6. Jones DJ, Fox MM, Babigian HM, Hutton HE: Epidemiology of anorexia nervosa in Monroe County, New York: 1960-1976. Psychosom Med 1980; 42:551-558

7. Hoek HW, Brook FG: Patterns of care of anorexia nervosa. J Psychiatr Res 1985; 19:155-160

8. Szmukler GI: The epidemiology of anorexia nervosa and bulimia. J Psychiatr Res 1985; 19:143-153

9. Williams P, King M: The "epidemic" of anorexia nervosa: another medical myth? Lancet 1987; 1:205-207

10. Nielsen S: The epidemiology of anorexia nervosa in Denmark from 1973 to 1987: a nationwide register study of psychiatric admission. Acta Psychiatr Scand 1990; 81:507-514

11. Møller-Madsen S, Nystrup J: Incidence of anorexia nervosa in Denmark. Acta Psychiatr Scand 1992; 86:197-200

12. Joergensen J: The epidemiology of eating disorders in Fyn County, Denmark, 1977-1986. Acta Psychiatr Scand 1992; 85: 30-34

13. Theander S: Anorexia nervosa: a psychiatric investigation of 94 female patients. Acta Psychiatr Scand Suppl 1970; 214:1-194

14. Willi J, Giacometti G, Limacher B: Update on the epidemiology of anorexia nervosa in a defined region of Switzerland. Am J Psychiatry 1990; 147:1514-1517

15. Lucas AR, Beard CM, O'Fallon WM, Kurland LT: 50-Year trends in the incidence of anorexia nervosa in Rochester, Minn: a population-based study. Am J Psychiatry 1991; 148:917-922

16. Cullberg J, Engström-Lindberg M: Prevalence and incidence of eating disorders in a suburban area. Acta Psychiatr Scand 1988; 78:314-319

17. Hoek HW: The incidence and prevalence of anorexia nervosa and bulimia nervosa in primary care. Psychol Med 1991; 21: $455-460$ 
18. Bartelds AIM, Fracheboud J, Van der Zee J: The Dutch Sentinel Practice Network: Relevance for Public Health Policy. Utrecht, Netherlands Institute of Primary Health Care (NIVEL), 1989

19. Frome EL, Checkoway H: Use of Poisson regression models in estimating incidence rates and ratios. Am J Epidemiol 1985; 121: 309-323

20. Garrow J: Treat Obesity Seriously: A Clinical Manual. Edinburgh, Churchill Livingstone, 1981

21. Mattingly D, Bhanji S: The diagnosis of anorexia nervosa. J R Coll Physicians Lond 1982; 16:191-194

22. Bryant-Waugh RJ: Do doctors recognise eating disorders in children? Arch Dis Child 1992; 67:103-105

23. Welch SL, Fairburn CG: Sexual abuse and bulimia nervosa: three integrated case control comparisons. Am J Psychiatry 1994; 151 : 402-407
24. Whitaker A, Davies M, Shaffer D, Johnson J, Abrams S, Walsh BT, Kalikow K: The struggle to be thin: a survey of anorectic and bulimic symptoms in a non-referred adolescent population. Psychol Med 1989; 19:143-163

25. Dohrenwend BP, Dohrenwend BS: Psychiatric disorders in urban settings, in American Handbook of Psychiatry, 2nd ed, vol II. Edited by Caplan G; Arieti S, editor-in-chief. New York, Basic Books, 1974

26. Lewis G, David A, Andréasson S, Allebeck P: Schizophrenia and city life. Lancet $1992 ; 340: 137-140$

27. Crowell BA, George LK, Blazer D, Landerman R: Psychosocial risk factors and urban/rural differences in the prevalence of major depression. Br J Psychiatry 1986; 149:307-314

28. Andersen AE (ed): Males With Eating Disorders. New York, Brunner/Mazel, 1990 